GENE 06200

\title{
Expression in mammalian cells of a cloned gene encoding murine DNA methyltransferase
}

(Recombinant DNA; shuttle vector; cytosine methylation assay; CpG dinucleotides)

\author{
Andreas Czank ", Remo Häuselmann a*, Andrea W. Page”, Heinrich Leonhardt", Timothy H. Bestor ${ }^{\text {b }}$, Walter Schaffner ${ }^{\text {a }}$ \\ and Martin Hergersberg ${ }^{a}$
}

"Institut für Molekularbiologie II der Universität Zürich. Hönggerberg, 8093 Zürich (Switzerland) Tel. (41-1)377-4411; Fax (41-1)-371-4873,

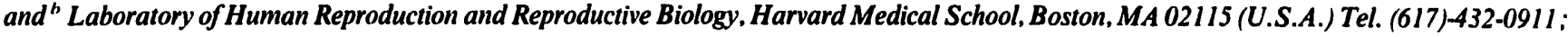
Fax (617)277-2732

Received by H.G. Zachau: 24 January 1991

Revised/Accepted: 15 August/16 August 1991

Received at publishers: 25 September 1991

\section{SUMMARY}

Mammalian DNA cytosine-5-methyltransferase (MTase, EC 2.1.1.37) is an essential component for establishing and maintaining cell-type specific methylation patterns in the genome. The cDNA for the murine enzyme was previously cloned in segments. We have reconstructed the entire gene, encoding a protein of 1517 amino acids, from a set of overlapping cDNA clones. We report the assembly of two expression constructs in bacterial/mammalian shuttle vectors. Transcription in the first construct (pEMT) is driven by the cytomegalovirus enhancer/promoter and encodes a fusion protein with 15 additional aa at the $\mathbf{N}$ terminus, while the second construct ( $\mathrm{pJMT}$ ) is driven by the simian virus 40 early promoter/enhancer upstream from the natural ATG codon. Immunofluorescence microscopy and immunoblot analysis have shown that both constructs direct the synthesis of MTase in COS-1 cells. Enzyme activity in whole-cell lysates of transfected COS-1 cells transfected with pFMT and pJMT are on average tenfold and fivefold higher than in controls, respectively. The specific activities of the recombinant and endogenous mouse-cell enzyme are similar. These expression constructs will be of use in studies of DNA methylation in mammals.

\section{INTRODUCTION}

A fraction of the cytosines in the dinucleotide 5'-CpG-3' are methylated in most eukaryotic genomes. The pattern of cytosine methylation is characteristic for each cell type and developmental stage. Changes in the

Correspondence to: Dr. W. Schaffner, Institut für Molekularbiologie II der Universität Zürich, Hönggerberg, 8093 Zürich (Switzerland)

Tel. (41-1)-377-3407 or -3404; Fax (41-1)371-4873.

* Present address: Pharmakologisches Institut der Universität Zürich, Gloriastrasse 32, 8006 Zürich (Switzerland)

Tel. (41-1)-257-2688.

A.C. and R.H. contributed equally to this work
CpG methylation are associated with activation of gene transcription, $\mathrm{X}$-chromosome inactivation and genomic imprinting (for recent reviews, see Bestor, 1990; Cedar, 1988; Hergersberg, 1991).

Thus far, MTases isolated from mammalian tissue or cell

Abbreviations: aa, amino acid(s); Ap, ampicillin; bp, base pair(s); CMV, cytomegalovirus; COS-1, CV-1 cells stably transiormed with the large SV40 T antigen; DMEM, Dulbecco's modified Eagle's meciium; DTT, dithiothreitol; FITC, fluorescein isothiocyanate; IVS, intervening sequence (intron); kb, kilobase(s) or $1000 \mathrm{bp}$; MEL, murine erythroleukemia cells; MTase, DNA cytosine-5-methyltransferase; ORF, open reading frame; ori, origin of DNA replication; PMSF, phenylmethylsulfonyl fluoride; SV40, simian virus 40; Tk, herpes simplex virus thymidine kinase; $t k$, gene encoding Tk. 

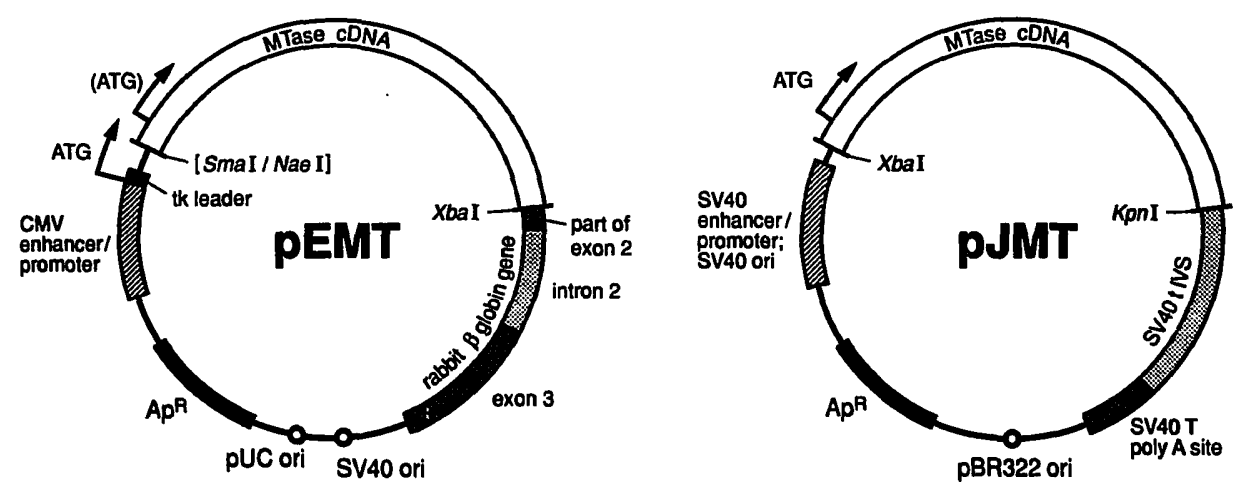

Fig. I. Schematic view of the MTase-encoding gene constructs in the expression vectors. The two expression vectors pEVRF0 (Matthias et al., 1989) and pJ3 $\Omega$ (Morgenstern et al., 1990) into which the MTase cDNA was cloned (the resulting constructs are pEMT and pJMT, respectively) are similar in concept, but use diferent fusctional elements. To stabilize the transcripts, both have, at the 3 ' end of the insert, an adjacent sequence with an intron and a transcriptional termination signal. Viral promoters that are active in many cell lines direct transcription, using the insert's natural ATG in pJ $3 \Omega$, whereas pEVRFU uses the ATG of the herpesvirus $t k$ leader sequence, resulting in a 15 additional aa. Plasmid pEVRF0 contains elements from pSP65, $\mathrm{pJ} 3 \Omega$ from $\mathrm{pBR} 322$. The figure is not to scait.



Fig. 2. Expression of the MTase cDNA in mammalian cells. The average MTase activity (height of the bars and the activity values) increase after transfection with the cDNA expression vectors pEMT and pJMT is shown, as compared to the values of DNA MTase activity in the control transfections with vectors pEVRF0 or POEVI, which were standardized to 1. All values are corrected for differences in protein concentration. Methods. Transfections were done according to de Villiers and Schaffner (1983) (DEAE dextran transfection) and Kingston et al. (1987) (Ca phosphate transfection), using 10 or $20 \mu \mathrm{g}$ of plasmid DNA in a transfection. Both transfection methods resulted in a similar increase in MTase enzyme activity. Protein concentration was determined by the Bio-Rad protein assay using bovine serum albumin as standard (Bradford, 1976). MTase extraction was performed as described (Bestor and Ingram, 1983). The cells were harvested $48 \mathrm{~h}$ after transfection and nuclear extracts were prepared. The nuclear extracts were dialyzed against $20 \mathrm{mM}$ Tris $\cdot \mathbf{H C l}$ $\mathrm{pH} 7.4$ for $2 \mathrm{~h}$ at $4^{\circ} \mathrm{C}$. Batch absorption/elution or column chromatography with DEAE Sephacel (pre-equilibrated with $20 \mathrm{mM}$ Tris $\cdot \mathrm{HCl}$ pH 7.4/0.5 mM DTT/0.2 mM PMSF) was used for partial purification of the MTase. Eluted by a gradient of $0.0-0.4 \mathrm{M} \mathrm{NaCl}, 10-\mu$ l aliquots of all fractions (five for batch elutions with different salt concentrations, 25-35 for the column eluate) were assayed for MTase enzyme activity and the values of each fraction were summed up relative to its volume. The transfer of radioactive methyl groups from $S$-adenosyl-[ ${ }^{3} \mathrm{H}$-methyl]-Lmethionine (Amersham) to poly(dIdC) (Pharmacia) was measured as described (Bestor and Ingram, 1983). To control for protein methylation, an aliquot of all fractions was added to assay mixtures with and without acceptor DNA and the latter value subtracted from the former. COS cells cultures have been large proteins of a size between 135 and $190 \mathrm{kDa}$, the smaller forms probably being proteolytic clipping forms of the 190-kDa protein (Pfeifer et al., 1983; Pfeifer and Drahovsky, 1986). The cDNA sequence reveals a clear homology between the C-terminal region and bacterial MTases (Bestor et al., 1988). In the N-terminal region a 'zinc finger' motif has been identified, which may be responsible for nonspecific DNA binding similar to the examples discussed by Berg (1990). No further homologies between the MTase and other known proteins were found (Bestor et al., 1988; Pósfai et al., 1989). MTases show a substrate preference for hemimethylated vs. unmethylated DNA, which is expected from the necessity to maintain methylated CpG sites over many cell generations. From the observation that cell types with different methylation patterns contain indistinguishable forms of MTase, it seems that the generation of differences in genome methylation is not an inherent property of the enzyme, but is achieved by other mechanisms.

We have overproduced the murine MTase protein in mammalian cells, which is a crucial step towards functional dissection of the enzymatic activity and its regulation, and a starting point to elucidate the function(s) of DNA methylation.

Here we report the assembly and propagation of the complete cDNA of the murine MTase-encoding gene in two mammalian expression vectors in Escherichia coli, as well as its introduction and expression in mammalian cells.

were cultured in DMEM supplemented with $2.5 \%$ fetal calf serum $/ 2.5 \%$ newborn calf serum $/ 2 \mathrm{mM}$ glutamine and antibiotics, at $\mathrm{CO}_{2}(5 \%) 37^{\circ} \mathrm{C}$. Cell culture products were from Gibco and Amimed. ${ }^{*}$ Transfections with the vector pEVRF0 or pOEV1 were standardized to 1 . Height of the bars corresponds to activation values. 


\section{EXPERIMENTAL AND DISCUSSION}

(a) Construction of the MTase expression vectors

The complete MTast: cDNA was assembled from two subclones containing overlapping fragments of 1.9 and
$3.8 \mathrm{~kb}$. The resulting $5.6 \mathrm{-kb}$ fragment, containing the 4551-bp ORF, was cloned into the expression vector pEVRF0 (Matthias et al., 1989; EMBL accession No. of the MTase sequence is X14508). This vector contains the strong enhancer/promoter of the human CMV (Boshart


B
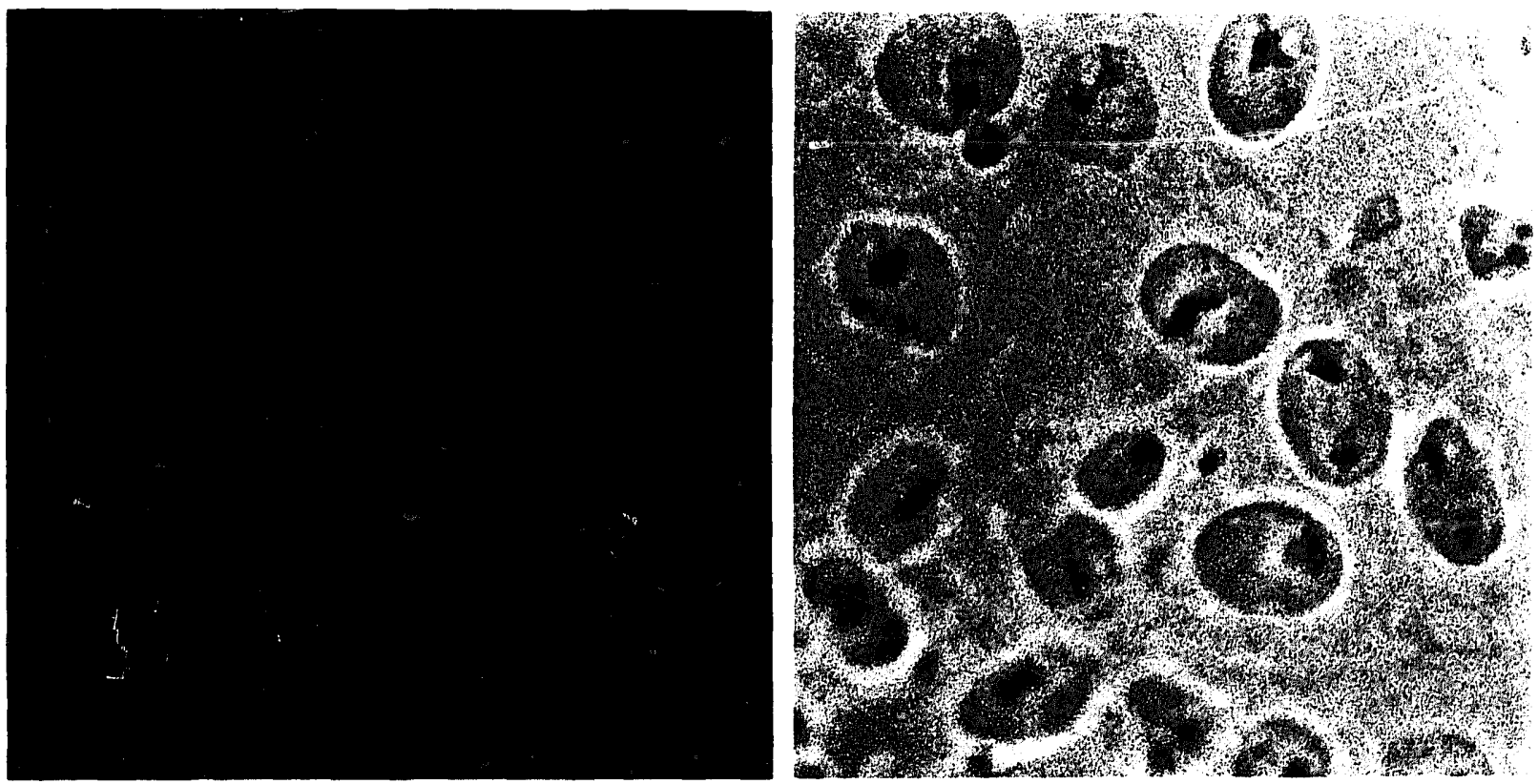

Fig. 3. MTase expression in COS cells monitored by immunofluorescence. (Panel A) COS cells were transfected with pEMT using the Ca-phosphate method (Kingston et al., 1987). $48 \mathrm{~h}$ later, the cells were rinsed, fixed with $4 \%$ formaldehyde, permeabilized with Triton X-100, and stained with a rabbit antiserum (dilution 1: 50) against MTase for 1-2 h, followed by a 1-h staining with a FITC-labelled goat anti-rabbit antibody. In this particular experiment, $5 \%$ of the nuclei showed bright nuclear fluorescence indicating strong expression of MTase. To the right of the immunofluorescence photographs in panels A and B phase contrast photographs were added to show the cell's substructures. (Panel B) As a control, the cells were treated as above, except that the vector (pEVRF0) without an insert was transfected. Of the over 5000 cells examined, none exhibited bright nuclear fluorescence. The cells are shown at higher magnification and display a faint nuclear fluorescence which is most likely due to endogenous MTase and/or unspecific staining. The rabbit antiserum to murine DNA MTase was produced by injection of a TrpE-MTase fusion protein produced in bacteria according to the protocol of Klempnauer and Sippel (1987). The fusion protein contained a portion of the MTase corresponding to aa 137-635. The immunofluorescence was done according to Harlow and Lane (1988). 
et al., 1985; Severne et al., 1988), which directs transcription from the ATG of the vector's herpesvirus $t k$ mRNA leader, resulting in an additional 15 aa at the $\mathbf{N}$ terminus. The resulting clone was named pEMT (Fig. 1). Sequencing revealed that the MTase ORF is in frame with the reading frame of the expression vector, and the sequenced part of pEMT showed no mismatches with the original cDNA clone.

A second expression construct, in which the MTase ORF starts at the natural MTase ATG, was made by inserting the full-length cDNA into the polylinker of the pJ $3 \Omega$ vector (Morgenstern and Land, 1990). Transcription is driven by the SV40 early enhancer/promoter. The resulting construct was named pJMT (Fig. 1).

\section{(b) Expression in COSi-1 cells}

COS- 1 cells constitutively express the large $T$ antigen of SV40 (Gluzman, 1981). Therefore, a plasmid construct like the expression vector pEVRF0 that includes an SV40 ori is amplified to high copy numbers in these cells (Mellon et al., 1981). This, in turn, results in high expression of the murine MTase gene. Cells were transfected with both pEMT and pJMT. Controls included untransfected cells, cells transfected with the 'empty' vector ( $\mathrm{pEVRF}$ ) or cells transfected with pOEV1, the same vector with an unrelated insert, namely the cDNA of the transcription factor Oct-2A (Muller et al., 1988). Activity assays and parallel immunoblot analysis were performed to compare the size and the specific activity of the recombinant and endogenous MEL cell MTase. COS- 1 cells were harvested $40 \mathrm{~h}$ after transfection, extracted and rates of transfer of radioactive methyl groups from $S$-adenosyl-[ ${ }^{3} \mathrm{H}$-methyl]-L-methionine to DNA were measured (Bestor and Ingram, 1983). Fig. 2 shows that whole-cell extracts from COS- 1 cells transfected with pEMT and pJMT had an average of $10.3( \pm 5.4)$ and 5.0 ( \pm 2.3 )-fold higher specific activity, respectively, compared to extracts from cells transfected with the control vectors alone.

MTase expression was directly demonstraled by immunofluorescence (Fig. 3). The nuclei of 5-20\% of the transfected cells were brightly stained in different experiments. Control experiments showed that the endogenous monkey MTase protein was only weakly reactive with the antimurine MTase antiserum and that intense staining depended on the expression of murine MTase coding sequences (not shown). Overexpression of the Oct-2A control had no effect on the MTase activity; activity in extracts from these cells was undistinguishable from activity in extracts of cells transfected with the vector alone (Fig. 2).

Immunoblot analysis showed that samples of extracts containing equal amounts of MTase enzyme activity from MEL cells and COS cells transfected with the MTase expression vectors produce bands of comparable intensity.
Thus we conclude that the recombinant MTase protein produced in COS-1 cells and the normal MEL cell enzyme have similar specific activities (Fig. 4). RNA extracts from both transfected and mock-transfected cells were analyzed by a dot blot (using radioactively labelled MTase cDNA as probe). mRNA from pEMT transfected cells yielded a ten-

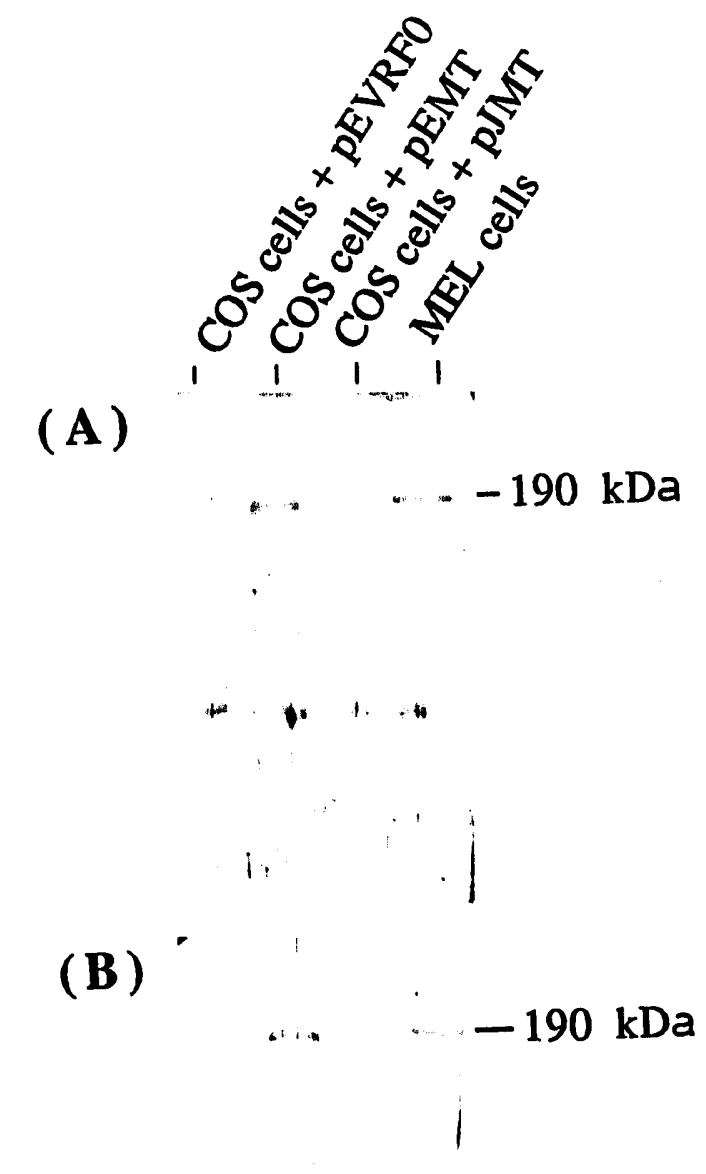

Fig. 4. Immunoblot analysis of recombinant and internal MEL cell MTase. To establish the identity and comparing the specific activities of the recombinant and the internal MEL cell MTase, whole-cell extracts were analyzed by Western blot. (Panel A) The recombinant and the internal MTase proteins have the same size. The proteins produced in COS- 1 cells migrate slightly faster than the MEL cell enzyme, probably due to differential post-translational modification. (Panel B) To compare the specific activity, portions of extracts were normalized with respect to the activity of MTase from MEL cells. The bands are of comparable intensity, which shows that the recombinant MTase and the endogenous MEL cell enzyme have similar specific activities. The pEVRF0 control contained an amount of total protein equal to that in the pEMT lane. From MEL cells, and COS- 1 cells transfected with pEMT, pJMT and pEVRF0 (as control) whole-cell lysates were made (Bestor and Ingram, 1983 ), using one tenth of an $80 \%$ confluent $100-\mathrm{mm}$ dish. The extracts were fractionated by $0.1 \%$ SDS-8\% PAGE and electroblotted onto nitrocellulose $\left(5 \mathrm{~mA} / \mathrm{cm}^{2} ; 30 \mathrm{~min}\right)$. The filter was blocked for $2 \mathrm{~h}$ with $5 \%$ dry milk and the blots probed with the anti-MTase antiserum (dilution 1:15000). As a second antibody an alkaline phosphatase-labelled goat anti-rabbit IgG (dilution 1:1500; Tago or Hyclone) was used and the colour development done according to the supplier's instructions. 
to twenty-fold higher signal compared to the mock-transfected ones (data not shown), which is consistent with the data above.

\section{(c) Conclusions and discussion}

We have assembled two constructs which direct the overexpression of full-length MTase cDNA at high levels in COS-1 cells. A five- to ten-fold increased MTase activity in COS-1 cells is certainly significant, but may not be overly impressive at first sight. However, in our transient expression assay only a fraction, typically about $10 \%$, of all the COS-1 cells are transfected with DNA. We therefore estimate that the increase in MTase activity in successfully transfected cells is about 100 -fold above the endogenous level (Fig. 2). The results presented in Fig. 2 also show that the 15-aa extension of the Tk leader sequence at the $\mathbf{N}$ terminus of the MTase protein produced from the pEMT vector does not interfere with MTase function. Since only low amounts of MTase are produced in cells and tissues and the enzyme is difficult to purify, progress in biochemical studies of this enzyme has been slow. The expression constructs described here should provide an advantageous tool for studies of the MTase enzyme and its function.

\section{ACKNOWLEDGEMENTS}

We are indebted to Drs. David Arnosti and Keith Harshman for critical reading of the manuscript and to Vreni Kurer and Fritz Ochsenbein for helping with the photography. M.H. was supported by an EMBO long-term postdoctoral fellowship.

\section{REFERENCES}

Berg, J.M.: Zinc fingers and other mental-binding domains. J. Biol. Chem. 265 (1990) 6513-6516.

Bestor, T.: DNA methylation: evolution of a bacterial immune function into a regulator of gene expression and genome structure in higher eukaryotes. Phil. Trans. R. Soc. Lond. B 326 (1990) 179-187.

Bestor, T. and Ingram, V.M.: Two DNA methyltransferases from murine erythroleukemia cells: purification, sequence specificity, and mode of interaction with DNA. Proc. Natl. Acad. Sci. USA 80 (1983) $5559-5563$.

Bestor, T., Laudano, A., Mattaliano, R. and Ingram, V.: Cloning ano sequencing of a cDNA encoding DNA methyltransferase of mouse cells. J. Mol. Biol. 203 (1988) 971-983.

Boshart, M., Weber, F., Jahn, G., Dorsch-Häsler, K., Fleckenstein, B. and Schaffner, W.: A very strong enhancer is located upstream of an immediate early gene of human cytomegalovirus. Cell 41 (1985) 521-530.

Bradford, M.M.: A rapid and sensitive method for the quantitation of microgram quantities of protein utilizing the principle of protein-dye binding. Anal. Biochem. 72 (1976) 248-254.

Cedar, H.: DNA methylation and gene activity. Cell 53 (1988) 3-4.

de Villiers, J. and Schaffner, W.: Transcriptional 'enhancers' from papovaviruses as components of eucaryotic gene expression vectors. In: Flavell, R.A. (Ed.), Techniques in Nucleic Acid Biochemistry, Vol. B5. Elsevier, Shannon, 1983, pp. 1-20.

Gluzman, Y.: SV40-transformed simian cells support the replication of early SV40 mutants. Cell 23 (1981) 175-182.

Harlow, E. and Lane, D. (Eds.): Antibodies. A Laboratory Manual. Cold Spring Harbor Laboratory, Cold Spring Harbor, NY, 1988, pp. 386, 392.

Hergersberg, M.: Biological aspects of cytosine methylation in eukaryotic cells. Experientia (1991) in press.

Kingston, R.E., Chen, C.A. and Okayama, H.: Transfection of DNA into eukaryotic cells. In: Ausubel, F.M., Brent, R., Kingston, R.E., Moore, D.D., Seidman, J.G., Smith, J.A. and Struhl, K. (Eds.), Current Protocols in Molecular Biology. Greene/Wiley Interscience, New York, 1987, pp. 9.1.1-9.1.9.

Klempnauer, K.H. and Sippel, A.E.: The highly conserved amino-teriainal region of the protein encoded by the v-myb oncogene functions as a DNA-binding domain. EMBO J. 6 (1987) 2719-2725.

Matthias, P., Müller, M.M., Schreiber, E., Rusconi, S. and Schaffner, W.: Eucaryotic expression vectors for the analysis of mutant proteins. Nucleic Acids Res. 17 (1989) 6418.

Mellon, P., Parker, V., Gluzman, Y. and Maniatis, T.: Identification of DNA sequences required for transcription of the human $\alpha 1$-globin gene in a new SV40 host-vector system. Cell 27 (1981) 279-288.

Morgenstern, J.P. and Land, H.: A series of mammalian expression vector and characterisation of their expression of a reporter gene in stably and transiently transfected cells. Nucleic Acids Res. 18 (1990) 1068.

Müller, M.M., Ruppert, S., Schaffner, W. and Matthias, P.: A cloned octamer transcription factor stimulates transcription from lymphoidspecific promoters in non-B cells. Nature 336 (1988) 544-55"

Pfeifer, G.P., Grünwald, S., Boehm, T.L.J. and Drahovsky, D.: Isolation and characterisation of DNA cytosine 5-methyltransferase from human placenta. Biochim. Biophys. Acta 740 (1983) 323-330.

Pfeifer, G.P. and Drahovsky, D.: DNA methyltransterase polypeptides in mouse and human cells. Biochim. Biophys. Acta 868 (1986) 238-242.

Pósfai, J., Bhagwat, A.S., Pósfai, G. and Roberts, R.J.: Predictive motifs derived from cytosine methyltransferases. Nucleic Acids Res. 17 (1989) 2421-2435.

Severne, Y., Wieland, S., Schaffner, W. and Rusconi, S.: Metal binding 'finger' structures in the glucocorticoid receptor defined by site-directed mutagenesis. EMBO J. 7 (1988) 2503-2508. 